Research Article

\title{
Representative Stress-Strain Curve by Spherical Indentation on Elastic-Plastic Materials
}

\author{
Chao Chang $\mathbb{D}^{1},{ }^{1}$ M. A. Garrido, ${ }^{2}$ J. Ruiz-Hervias, ${ }^{3}$ Zhu Zhang, ${ }^{1}$ and Le-le Zhang ${ }^{4}$ \\ ${ }^{1}$ School of Applied Science, Taiyuan University of Science and Technology, Taiyuan 030024, China \\ ${ }^{2}$ Departamento de Tecnología Química y Ambiental, Tecnología Química y Energética y Tecnología Mecánica, Escuela Superior \\ de Ciencias Experimentales y Tecnología, Universidad Rey Juan Carlos, c/Tulipán s/n Móstoles, Madrid, Spain \\ ${ }^{3}$ Departamento de Ciencia de Materiales, UPM, E.T.S.I. Caminos, Canales y Puertos, $c /$ Professor Aranguren $s / n$, \\ 28040 Madrid, Spain \\ ${ }^{4}$ School of Mechanical, Electronic and Control Engineering, Beijing Jiaotong University, Beijing 100044, China
}

Correspondence should be addressed to Chao Chang; chao_chang_tyust@163.com

Received 12 August 2017; Revised 4 December 2017; Accepted 25 December 2017; Published 26 February 2018

Academic Editor: Baozhong Sun

Copyright (c) 2018 Chao Chang et al. This is an open access article distributed under the Creative Commons Attribution License, which permits unrestricted use, distribution, and reproduction in any medium, provided the original work is properly cited.

Tensile stress-strain curve of metallic materials can be determined by the representative stress-strain curve from the spherical indentation. Tabor empirically determined the stress constraint factor (stress CF), $\psi$, and strain constraint factor (strain CF), $\beta$, but the choice of value for $\psi$ and $\beta$ is still under discussion. In this study, a new insight into the relationship between constraint factors of stress and strain is analytically described based on the formation of Tabor's equation. Experiment tests were performed to evaluate these constraint factors. From the results, representative stress-strain curves using a proposed strain constraint factor can fit better with nominal stress-strain curve than those using Tabor's constraint factors.

\section{Introduction}

The instrumented indentation technique consists of applying load to the sample by means of an indenter of known geometry, while the applied load and the penetration depth of the indenter are recorded simultaneously during a loading and unloading cycle. The load-penetration data can be used to determine the mechanical properties of the material without having to image the residual impression left on the material's surface. Consequently, this technique can be applied at different scales, from macro to nano. The main mechanical properties measured by this technique are Young's modulus, $E$, and hardness, $H$, by sharp indenter $[1,2]$. This methodology could also measure material properties like yield stress, creep property, and fracture [3-6]. However, these properties are not sufficient to characterize a material. The present work focuses on the methodology to determine the stress-strain curve of metallic materials by the depth-sensing indentation technique using a spherical indenter. Sharp indenters like Berkovich or Vicker are characterized by inducing a constant strain on the indented materials. This deformation depends on the indenter angle. Consequently, if the complete stressstrain curve is needed, the sharp indenters are not the best option because they would give only a point of such curve. This is the reason why the interest for the spherical indenter has been recently grown. The experimental curve from spherical indenter provides more information, as this type of indenter has a smooth transition from elastic to elastic-plastic contact [7].

There were many methodologies for the extraction of flow stress with the spherical indentation technique. One category belongs to mathematical and numerical methods (e.g., dimensionless method and artificial neural network (ANN)). After running many finite element models within certain range of material properties, the indentation curves, contact stiffness, or the work of loading-unloading is obtained, which are verified by corresponding experimental indentation and establish a relation between the indentation characteristics and material properties [8-15]. However, these methods consume great computational effort. The unique solution of these methods is still under discussion $[16,17]$. Moreover, the contamination in the experimental 
process like deficient indenter tip, roughness of specimen, or intrinsic noise of instrument machine is not considered in these methods.

Another category is to determine the material parameters from spherical indentation by defining the representative strain and stress. Regarding the experimental measurements, these methods are more convenient and can be used directly. Following the work of Meyer [18], Tabor defined the representative indentation strain, $\varepsilon_{r}$, at contact edge of the spherical indenter as $\varepsilon_{r}=0.2(a / R)$, where the values of $\psi$ and $\beta$ empirically were determined as 2.8 and 0.2 , respectively, based on the quantity of tensile tests on common metals [19]. Most of the researchers agreed with Tabor's indentation strain but emerge with the controversy on the choice of values of $\psi$ and $\beta[5,20-25]$. Richmond et al. [21] predicted that the mean pressure was approximately equal to 3 times the yield stress and that the representative strain was approximately equal to 0.32 times the impression to ball diameter ratio. Herbert et al. [23] had used higher stress constraint factor of 3.7 to obtain the representative stress-strain curve for $\mathrm{Al} \mathrm{6061-T6} \mathrm{by}$ spherical indentation with a radius of $385 \mathrm{~nm}$. Based on the work of Matthews [22], Tirupataiah and Sundararajan [26] derived an expression for the indentation stress factor, $\psi$, which was related with hardening exponent, n. Similarly, Yetna N'Jock et al. [27] determined the tensile property by spherical indentation by simply using the expression of mean pressure to stress ratio as a function of hardening exponent which was derived from the FEM works of Taljat et al. [28]. Using the representative strain under spherical indentation, Ahn and Kwon [29] developed a shear strain definition by differentiating the displacement in the depth direction, in which the ratio of mean pressure to representative stress was equal to 3 in the fully plastic period by conducting instrumented spherical indentation on the steel specimen. By carrying out extensive forward analyses in FEM, Xu and Chen [24] using the indentation strain by Ahn and Kwon [29] found the indentation stress constraint factors, $\psi$, depended almost linearly on hardening exponent, $n$. Additionally, the corresponding indentation strain constraint factor $\beta$ depended on both $n$ and the ratio of Young's modulus to yield stress. Milman et al. [30] assumed that the fully plastic zone beneath indenter was incompressible and proposed a new representative strain which was related with contact radius and contact depth. Fu et al. [31] used the Milman representative strain [30] and proposed a novel iterative process to determine the tensile stress-strain curve. Recently, Kalidindi et al. $[32,33]$ argued that the definition of indentation strain as $a / R$ lacks any physical interpretation as a measure of strain and proposed a new definition of the indentation strain consistent with the Hertz theory, which was evaluated from several FEM simulations as well as from the analysis of experimental measurements.

From the literature, there is not uniform agreement among investigations concerning the representative stress and strain equations and also the values for the factors involved in the expressions. The main purpose of the present investigation is to systematically study Tabor's indentation strain and propose the possibility to develop an analytical procedure to extract the stress-strain curve using experimental data from spherical indentations, which would be comparable to that obtained from a uniaxial test (i.e., tensile test).

\section{Theoretical Background}

2.1. The Analytical Relationship between Stress and Strain Factors. In 1908, Meyer had found that for many materials, the mean pressure increased with $a / R$ according to the simple power law [18]

$$
p_{m}=k\left(\frac{a}{R}\right)^{m}
$$

in which $p_{m}$ is the mean pressure, $a / R$ is the ratio of indentation radius to the ball radius, and $m$ is the Meyer index.

In (1), $k$ and $m$ are constants. The Meyer equation was verified by further experimental studies [19, 34], and they suggested that the Meyer index, $m$, was related with the hardening exponent, $n$. Following Meyer's work, Tabor had proposed the concept of representative strain or stress, by which the mean pressure in Meyer's equation and the $a / R$ ratio can be converted into the true stress-strain curve. He assumed that the mean pressure, $p_{m}$, at the fully plastic regime was proportional to the representative stress, $\sigma_{r}$, and the impression radius was proportional to the corresponding representative strain, $\varepsilon_{r}$. Consequently, the representative stress and strain can be expressed as [19]

$$
\begin{gathered}
\varepsilon_{r}=\beta \frac{a}{R}, \\
\sigma_{r}=\frac{F}{\psi \pi a^{2}},
\end{gathered}
$$

where $\beta$ is the indentation strain constraint factor, $a$ is the contact radius, $R$ is the radius of the indenter, $\psi$ is the indentation stress constraint factor, and $F$ is the indentation load.

Tabor determined the parameters $\beta$ and $\psi$ from empirically experimental data from spherical indentations under the fully plasticity regime. Generally, the indentation strain constraint factor $\beta$ is considered to be equal to 0.2 , and the indentation stress constraint factor $\psi$ ranges from 2.8 to 3.2 [35]. It should be emphasized that the representative strain and stress defined by Tabor are an average value of the stress and strain states induced inside the material $[36,37]$. The stress and strain constraint factors allow us to establish equivalence between the stress-strain indentation curve and the corresponding one obtained from a uniaxial test. The true stress-strain curve from uniaxial tensile or compression test can be expressed as

$$
\begin{aligned}
& \sigma=E \varepsilon \text { for } \sigma \leq \sigma_{\mathrm{y}} \text { elastic regime, } \\
& \sigma=K \varepsilon^{n} \quad \text { for } \sigma \geq \sigma_{\mathrm{y}} \text { Hollomon's equation for plastic regime, }
\end{aligned}
$$

where $E$ is the elastic modulus, $\sigma_{y}$ is the yield' stress, $K$ is the strength coefficient, and $n$ is the strain-hardening exponent. 


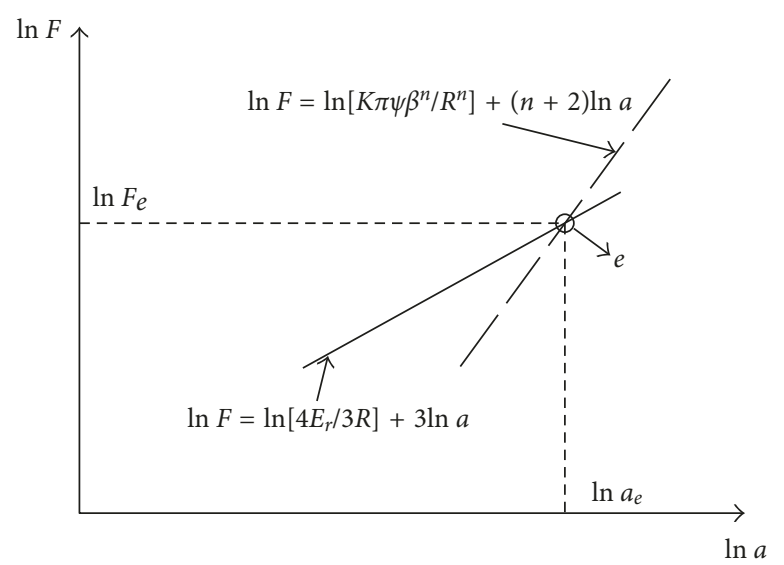

FIgURE 1: Equations (6) and (8) are in the same $\ln a-\ln F$ coordinate, and " $e$ " is the intersectional point.

TABLE 1: Mechanical properties of steel F114, brass, and Al alloy.

\begin{tabular}{lcccc}
\hline Materials & $E(\mathrm{GPa})$ & $\begin{array}{c}\text { Yield stress } \\
(\mathrm{MPa})\end{array}$ & $\begin{array}{c}\text { Hardening } \\
\text { exponent } n\end{array}$ & $\begin{array}{c}\text { Hardening exponent } \\
n \text { determined by indentation }\end{array}$ \\
\hline Steel F114 & 210 & 820 & 0.26 & 0.20 \\
Brass alloy & 100 & 320 & 0.15 & 0.19 \\
Al alloy & 70 & 220 & 0.1 & 0.15 \\
\hline
\end{tabular}

Substituting (2) into the Hollomon equation:

$$
\frac{F}{\psi \pi a^{2}}=K\left[\beta\left(\frac{a}{R}\right)\right]^{n}
$$

which represents the relationship between load, $F$, and the ratio of contact radius to indenter radius according to the power law at the fully plastic regime. Transforming (5) into the natural logarithm as

$$
\ln F=\ln \left[\frac{K \pi \psi \beta^{n}}{R^{n}}\right]+(n+2) \ln a .
$$

According to Hertz equation under the elastic regime, the loading and contact radius can be expressed as

$$
F=\frac{4 E_{r}}{3 R} a^{3},
$$

where $1 / E_{r}=\left(1-v^{2} / E\right)+\left(1-v_{i}^{2} / E_{i}\right), E, v$ and $E_{i}$ and $v_{i}$ are Young's modulus and Poisson's ratio for the bulk material to be measured and for the indenter, respectively.

Transforming (7) into the natural logarithm

$$
\ln F=\ln \left[\frac{4 E_{r}}{3 R}\right]+3 \ln a .
$$

Most metals have an $n$ value between 0.10 and 0.50 , plotting (6) and (8) in the $\ln a$ versus $\ln F$ coordinate, as shown in Figure 1. Inversely extending the two lines, there should be an intersectional point " $e$ " for different linear slopes.

Therefore, the representative strain and stress at the point " $e$ " can be expressed as

$$
\varepsilon_{r}=E \beta\left(\frac{a_{e}}{R}\right),
$$

$$
\sigma_{r}=\frac{F_{e}}{\psi \pi a_{e}^{e}} .
$$

Combining (9) and (10), we can obtain

$$
\psi * \beta=\frac{R}{\pi E} \cdot \frac{F_{e}}{a_{e}^{3}} .
$$

Comparing (10) with Hertz equation in (7), we can obtain

$$
\phi=\psi * \beta=\frac{4}{3 \pi} \cdot \frac{E_{r}}{E} .
$$

In (12), the product, $\phi$, of stress constraint factor $\psi$ and strain constraint factor $\beta$ is constant, which is dependent on the ratio of reduced Young's modulus $E_{r}$ to Young's modulus E. According to (12), the constraint factors can be determined if one of them is known.

It should be noted that Tabor's representative method is restricted within the fully plastic regime. In this study, the procedure assumes that elastic-plastic transition is negligible, and the relationship between stress and strain constraint factors is affirmed from Hertz's theory. In Kalidindi et al.'s [32, 33] work, they utilized indentation strain derived from the Hertz theory for extracting the full stress-strain curve of metallic materials. Similarly, we utilize the Hertz equation to study the relationship between the stress and strain constraint factors to extract the stress-strain curve based on Tabor's representative method. For the soft metallic materials with high $E / \sigma_{y}$ which has very short elasticplastic transition, the intersection point " $e$ " can be considered as a simplistic interpretation of the elastic-plastic transition regime. Especially, in experimental practice, when performing the nanoindentation test on the soft metallic 


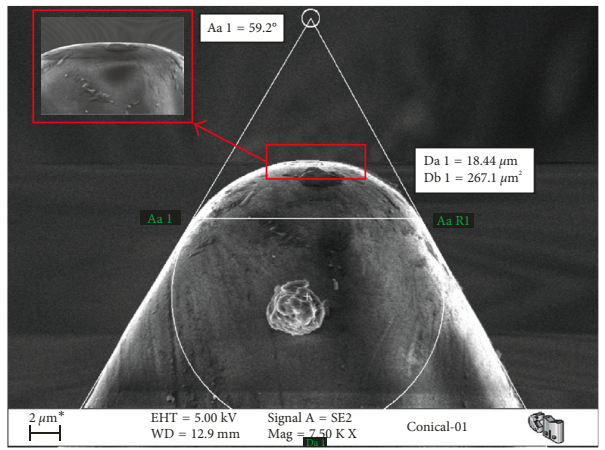

Figure 2: Scanning electron microscope image of a worn diamond conical-spherical indenter.

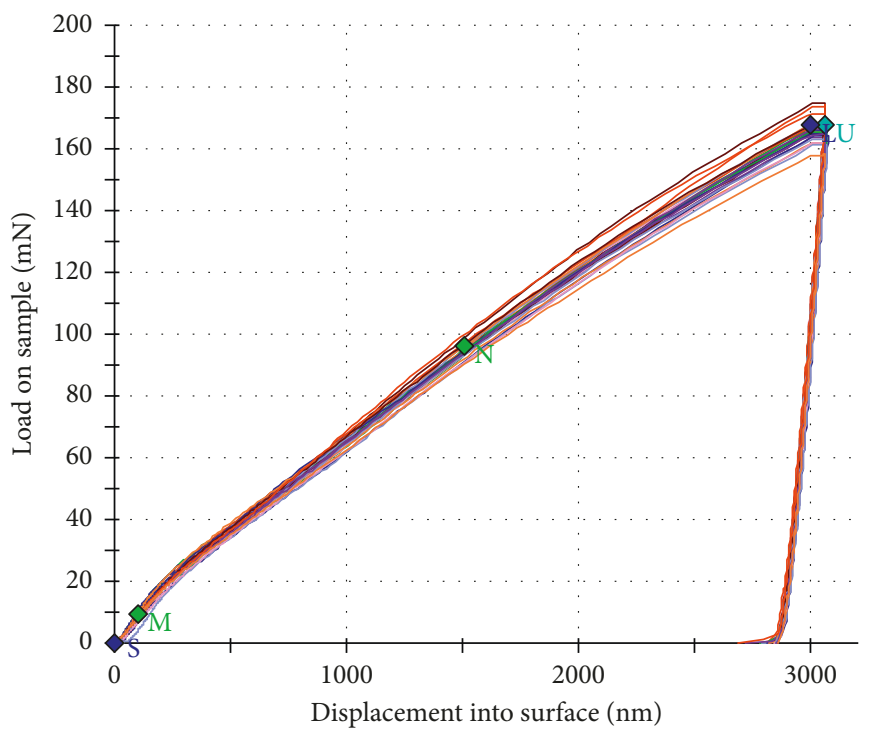

(a)

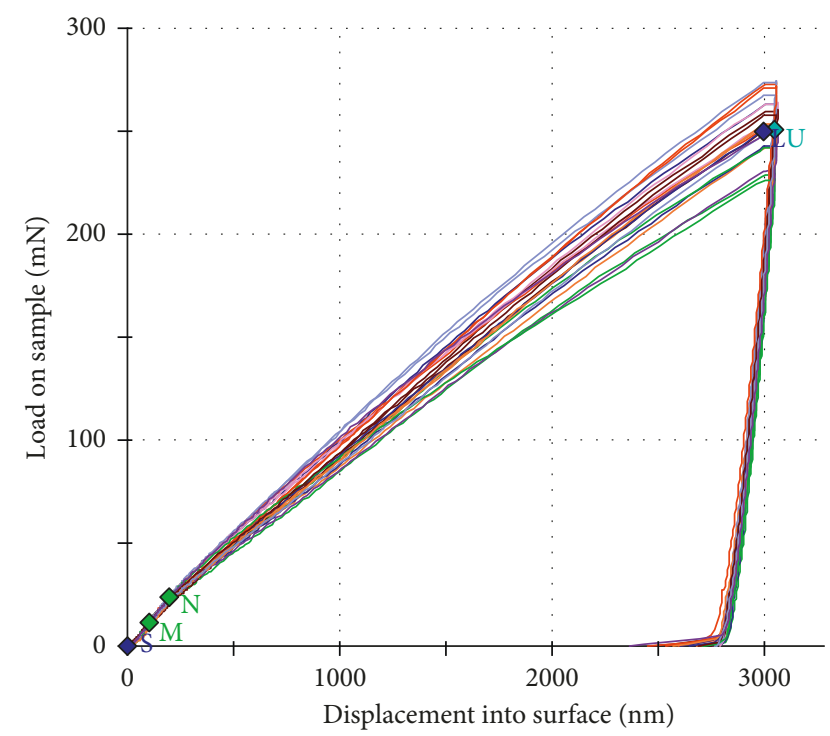

(b)

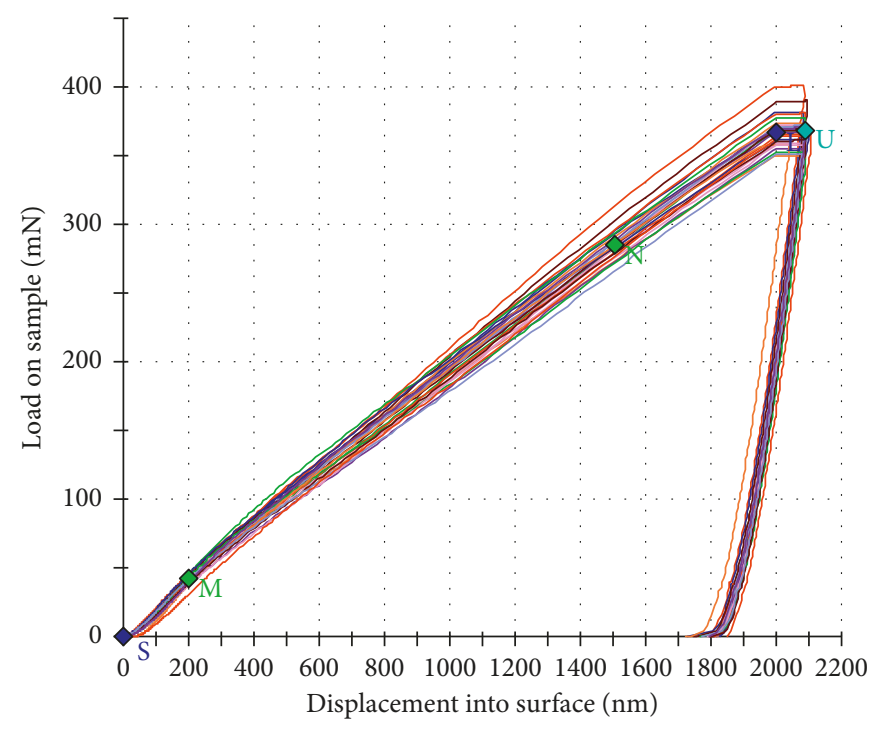

(c)

Figure 3: The load versus penetration depth curves for the three indented materials performed with spherical tip. (a) Al-1050, (b) brass, and (c) steel. 
materials, the penetration depth is very shallow to turn into the fully plastic regime. Consequently, this assumption is reasonable only for certain soft metallic materials. The experimental verification will be discussed later.

In Tabor's equation, the value of stress constraint factor is selected as 3 for common engineering metals in literature $[5,31,38]$. However, there are few studies on the analytical solution for the stress constraint factor. The indentation pressure is found to be proportional to the yield strength of the material by the constraint factor, $C$. The magnitude of the constraint factor has been found to be depended on the material properties, particularly on the ratio of the modulus to the yield stress. For most metals, $E / \sigma_{y}>100$, and the constraint factor is approximately equal to $3(C \approx 3)$ [19]. For polymers, the ratio of $E / \sigma_{y}<10$ and the constraint factor is less than 3 [39]. An approach to large strain plasticity problems in which the material is considered to behave in a plastic-elastic fashion, instead of as a plastic-rigid body, is applied to the axisymmetric blunt indenter. The ratio of the mean stress on the punch face to the uniaxial flow stress of the material (constraint factor $C$ ) is found to be 2.82 for an extensive specimen. However, it is shown that a small part of the punch face is elastically loaded, and if the loaded punch area is assumed to be equal to the size of the plastic impression, then the constraint factor to be used is 3 instead of 2.82. This is the value to be used in interpreting the ordinary Brinell test[40]. Additionally, Shield found the exact solution for the axisymmetric flat punch. He found a maximum pressure under the punch of $2.8 \sigma_{y}$ at the centre [41]. Von Mises yield conditions are more representative of engineering materials than Tresca. Although the slip line field theory can be applied to both types of yield conditions, Tresca is generally selected as it leads to a simpler equation that can be solved analytically. However, Von Mises yield can give up to $15.5 \%$ higher limit load value than Tresca, which would lead to $C=3.2$ [42]. Unlike the punch indenter, where the contact area is constant, the spherical one offers a gradual transition of the contact area. It could be assumed that the spherical indenter is corresponding to a punch indenter at a given penetration depth. As a result, the value of stress constraint factor is taken as 3.2 , which is consistent with the analytical solution by Shield [41]. As the product of stress and strain constraint factors is constant, according to (12), the strain constraint factor can be determined.

2.2. Estimation of Contact Radius. In order to calculate the representative stress and strain, an accurate measurement of the contact radius at defined indentation load would be obtained. In the case of a flat punch indenter, Sneddon's analysis proposed an estimation expression of contact radius, $a$ :

$$
a=\frac{S_{u}}{2 E_{r}}
$$

where $S_{u}$ is the contact stiffness.

From Eq. (13), the contanct radius and Young's modulus can be extracted from the initial unloading slope [43]. According to (13), the contact radius could be determined

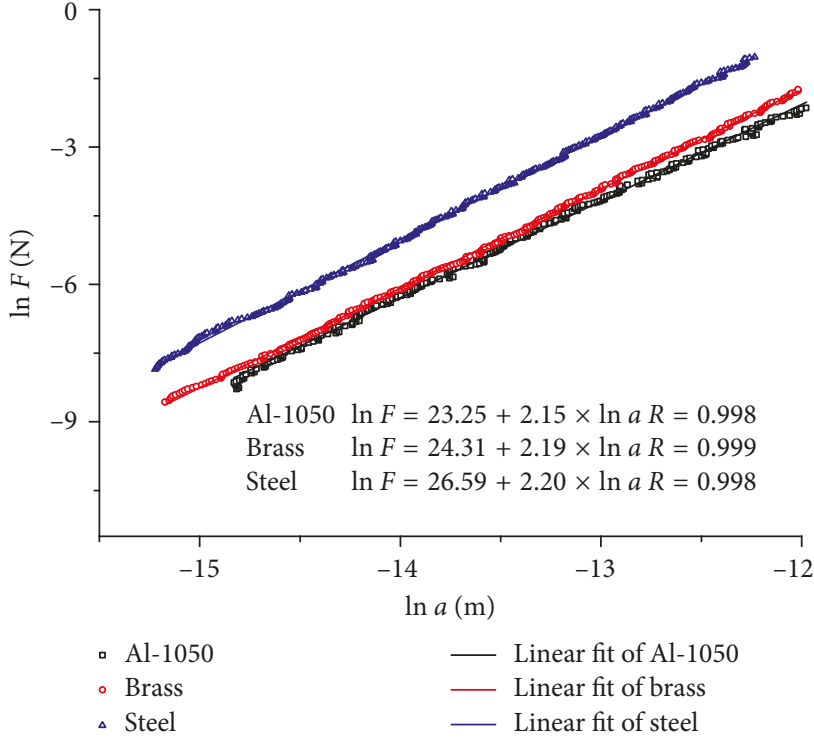

FIGURE 4: Linear regression of $\ln (F)$ versus $\ln (a)$ for the experimental materials.

when reduced Young's modulus and initial unloading slope of the unloading are known. The procedure to estimate the contact radius is consistent with Kalidindi et al.'s [32, 33] work.

\section{Experimental Procedure}

Depth-sensing indentation (DSI) tests were performed on a Nanoindenter XP (Nanoinstruments Innovation Center, MTS systems, TN, USA) by using the continuous stiffness measurement (CSM) methodology. A conical (angel $60^{\circ}$ ) spherical diamond indenter with a tip radius of $10 \mu \mathrm{m}$ was selected. To verify the radius of the indenter, scanning electron microscope was used to measure the radius of the tip before the test. Continuous loading and unloading cycles were conducted during the loading branch by imposing a small dynamic oscillation of $2 \mathrm{~nm}$ and $45 \mathrm{~Hz}$ on the displacement signal and measuring the amplitude and phase of the corresponding force $[44,45]$. Consequently, the contact stiffness was continuously measured as a function of the penetration depth during the experiment. A tip calibration procedure was carried out using the fused silica in accordance with the CSM methodology [46]. Prior to the indentation tests, the samples were ground and polished in two steps, using a mechanical polisher (Labopol-5, Struers, Copenhagen, Denmark) and finished with colloidal suspension of silica of $0.05 \mu \mathrm{m}$. A total of 25 indentations were performed in displacement control on three samples of commercial metallic alloys: a carbon steel (F-114), a brass alloy, and an aluminum alloy (Al-1050). The penetration depth was selected according to the grain size of indented materials: $3000 \mathrm{~nm}$ for Al-1050 and brass and $2000 \mathrm{~nm}$ for steel. The average of these curves is selected to determine the representative stress-strain curve by spherical indentation which can be comparable with the true stress-strain curve by tensile tests [3]. Tensile tests were also conducted 


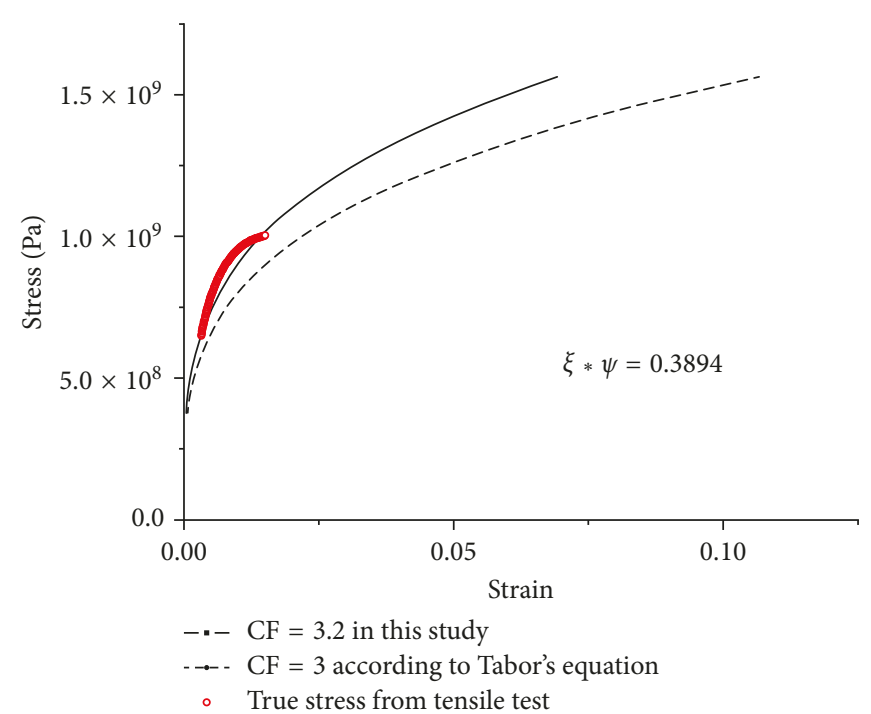

(a)

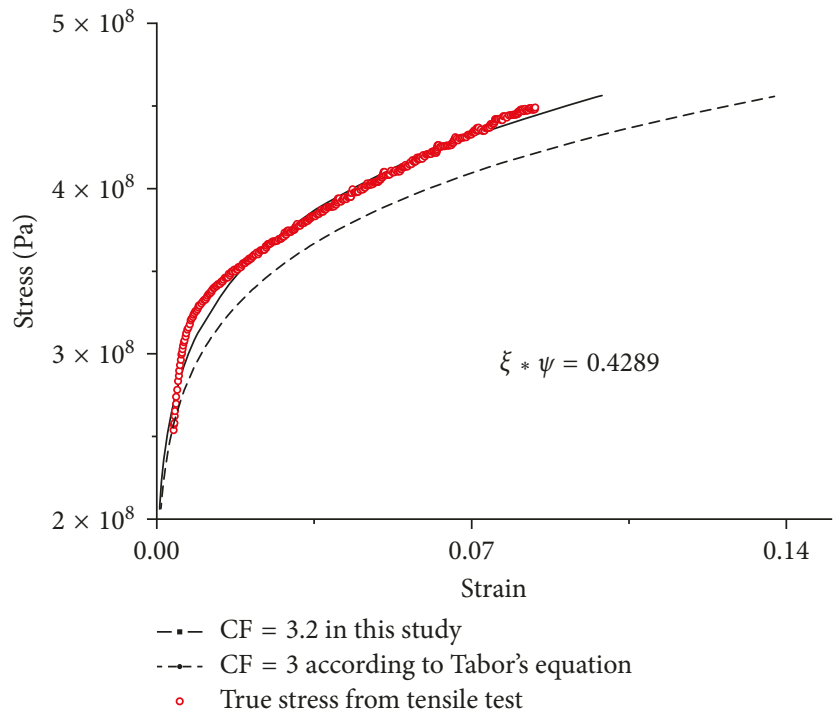

(b)

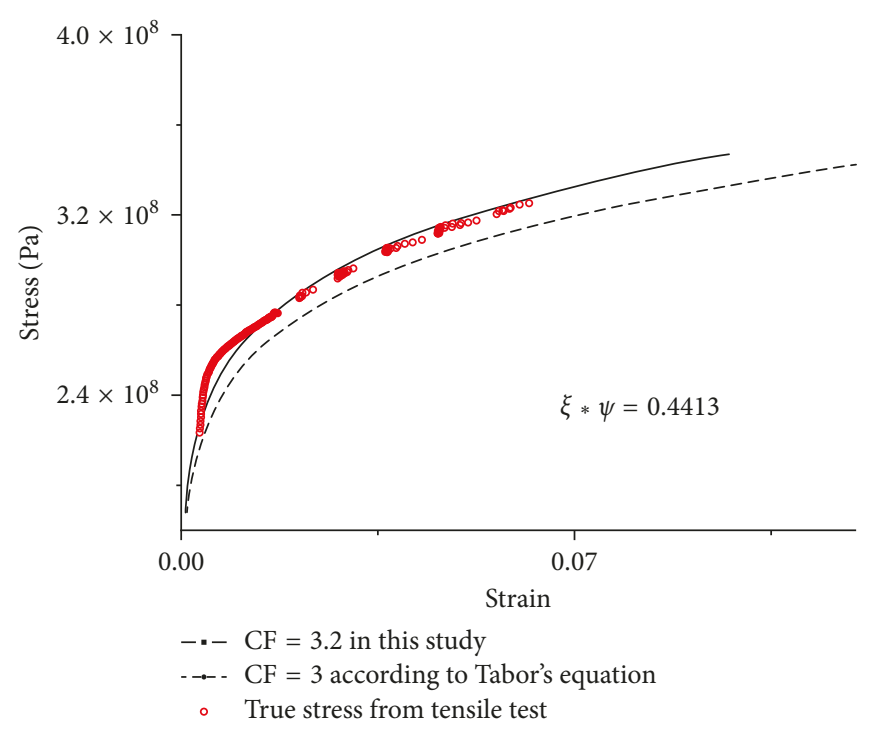

(c)

Figure 5: Curve plots for the representative stress-strain curve, Tabor's stress-strain curve, and the true stress-strain curve extracted from the tensile test. (a) Steel, (b) brass, and (c) aluminum.

on carbon steel (F-114), aluminum (Al-1050), and brass alloy samples. They were performed on a universal testing machine INSTRON 8501 with a $10 \mathrm{kN}$ load cell at room temperature under displacement control by using a displacement rate of $0.5 \mathrm{~mm} / \mathrm{min}$. Cylindrical specimens were machined according to the ASTM E8 standard [47]. The gage length was $12.5 \mathrm{~mm}$ for the samples.

\section{Results and Discussion}

In the tensile test, according to Hollomon's equation, the mechanical properties can be obtained from the stress-strain curve. Young's modulus, yield stress which is determined by means of the $0.2 \%$ strain rule, and the hardening exponent are shown in Table 1.
In order to check the radius of the spherical indenter, as shown in Figure 2, the best fitting spherical radius is found to be about $9.2 \mu \mathrm{m}$ from SEM measurement which is smaller than the nominal value. From the zoomed-in figure, the spherical indenter is worn at shallow penetration depth. In addition, inherent noise of continuous stiffness measurement and the roughness of specimen could scatter the representative stress-strain curve. Consequently, penetration depths less than $10 \mathrm{~nm}$ will not be utilized for the extraction of representative stress-strain curve.

Figure 3 shows the load versus penetration depth curves for the three indented materials performed with spherical tip. The indenter curves of brass exhibit considerable scatter, as the diameter of the indenter is comparative to the grain size of brass listed in Table 1. On the 
contrary, the indenter curves of steel and Al-1050 are much more reproducible for the multiple grain indents. The average of these curves is selected to determine the representative stress-strain curve. The procedure is consistent with the previous work [3].

When Young's modulus was confirmed by the tensile test and the contact stiffness can be measured by CSM, the contact radius can be estimated by Sneddon's equation [48]. It should be noted that, when using (13) to estimate the contact radius, Young's modulus is assumed as a constant during the indentation process $[32,49]$. Tabor had concluded that the slope of the linear regression is Meyer's index $m$ which is equal to $n+2$, where $n$ is the hardening exponent in the Hollomon equation. Figure 4 shows the linear regression of $\log (F)$ versus $\log (a)$ for the experimental materials. The values of hardening exponent from the slope are in good agreement with those obtained from the tensile experiments in Table 1.

In order to check the reasonable determination of constraint factors in Tabor's equation, the tensile stressstrain curves are compared with representative stress-strain curves from a different constraint factor $(\mathrm{CF})$ : stress $\mathrm{CF}=3.2$ in this study and stress $\mathrm{CF}=3$ according to Tabor's equation. In the experimental analysis, the value of stress constraint factor is taken as 3.2 , and the strain constraint factor is confirmed from (12). In order to verify the reasonability of the confirmation of constraint factors from experimental data, the representative stress-strain curve extracted from this study will be compared with the curve extracted from Tabor's method.

Figure 5 shows the plots for the indentation stress-strain curve using the constraint factors in the study, the indentation stress-strain curve using the constraint factors in Tabor's equation, and the true stress-strain curve extracted from the tensile test. The values of $E / \sigma_{y}$ for studied materials range from 250 to 320 . Although the initial part of the true stress-strain curve and the representative stress-strain curves shows little derivation between them, the representative stress-strain curve using the constraint factors in the study shows well agreement with the true stress-strain curve from the tensile test in the fully plastic regime. In this study, the product $\phi$ is attained from the intersection between the elastic regime and the fully plastic regime, in which the effect of the transition from the elastic to plastic regime is ignored. This assumption is only suitable for the soft metal which has short transition of the elastic-plastic regime, and the fully plastic regime is developed at shallow penetration depth. For metals with longer transition of the elastic-plastic regime, it can be seen that there is significant derivation in the initial part of the representative stress-strain curve regarding the true stress strain curve obtained from tensile test. As noted in literature $[32,33,50,51]$, it is very difficult to make a comparable stress-strain curve with the tensile test in the elastic-plastic transition by Tabor's representative method for the metallic materials with low $E / \sigma_{y}$. The FEM simulations also showed that representative stress-strain from Kalidindi's equation led to large deviation for some materials with large transition of the elastic-plastic regime [52]. However, in the study, utilizing the proposed procedure to determine the constraint factors could make a comparable stress-strain curve in the fully plastic regime.

\section{Conclusion}

The main conclusion in the paper can be summarized as follows:

(1) In this study, a new insight into the constraint factors in the formation of Tabor's equation is analytically described. The product $\phi$ of strain constraint factor $\beta$ and stress constraint factor $\psi$ is constant at the transition position between the elastic regime and fully plastic regime, which is related to Young's modulus of contact materials.

(2) An experimental procedure is performed to evaluate the analytical analysis for extracting the representative stress-strain curve from the spherical indentation. The representative stress-strain curve using the proposed constraint factors in the study shows well agreement with the nominal true stressstrain curve.

(3) Due to the drawback of the formation of Tabor's equation, the representative stress-strain curve derived from the Tabor's formation could have large deviation at the initial strain period for the materials with longer elastic-plastic transition. In future, formation of new representative strain for spherical indentation needs more study.

\section{Nomenclature}

$\begin{array}{ll}p_{m}: & \text { Mean pressure } \\ a: & \text { Contact radius } \\ R: & \text { Indenter radius } \\ m: & \text { Meyer's index } \\ k: & \text { Coefficient in Meyer's equation } \\ \varepsilon_{r}: & \text { Representative strain } \\ \sigma_{r}: & \text { Representative stress } \\ \beta: & \text { Indentation strain constraint factor } \\ \psi: & \text { Indentation stress constraint factor } \\ E: & \text { Elastic modulus } \\ \sigma_{y}: & \text { Yield stress } \\ K: & \text { Strength coefficient in Hollomon's equation } \\ n: & \text { Strain-hardening exponent } \\ \nu: & \text { Poisson's ratio } \\ \phi: & \text { Product of stress constraint factor } \psi \text { and strain } \\ & \text { constraint factor } \beta \\ e: & \text { Elastic component. }\end{array}$

\section{Conflicts of Interest}

The authors declare that they have no conflicts of interest regarding the publication of this paper.

\section{Acknowledgments}

This work was supported by the National Key R\&D Program of China (2016YFB1200403-A-02), the Natural Science Foundation of Shanxi Province (no. 201701D221008), the 
PhD Research Startup Foundation of Taiyuan University of Science and Technology (no. 201704), the Shanxi Province Science Foundation for Youths (no. 2015021021), and the National Science Foundation for Young Scientists of China (no. 11702182).

\section{References}

[1] M. F. Doerner and W. D. Nix, "A method for interpreting the data from depth-sensing indentation instruments," Journal of Materials Research, vol. 1, no. 4, pp. 601-609, 1986.

[2] W. C. Oliver and G. M. Pharr, "An improved technique for determining hardness and elastic modulus using load and displacement sensing indentation experiments," Journal of Materials Research, vol. 7, no. 6, pp. 1564-1583, 1992.

[3] J. Dean, J. Wheeler, and T. Clyne, "Use of quasi-static nanoindentation data to obtain stress-strain characteristics for metallic materials," Acta Materialia, vol. 58, no. 10, pp. 3613-3623, 2010.

[4] M. Á. Garrido, I. Giráldez, L. Ceballos, and J. Rodríguez, "On the possibility of estimating the fracture toughness of enamel," Dental Materials, vol. 30, no. 11, pp. 1224-1233, 2014.

[5] P. Haušild, A. Materna, and J. Nohava, "On the identification of stress-strain relation by instrumented indentation with spherical indenter," Materials \& Design, vol. 37, pp. 373-378, 2012.

[6] J. S. Field and M. V. Swain, "Determining the mechanical properties of small volumes of material from submicrometer spherical indentations," Journal of Materials Research, vol. 10, no. 1, pp. 101-112, 1995.

[7] A. C. Fischer-Cripps, Factors Affecting Nanoindentation Test Data, Springer, Berlin, Germany, 2000.

[8] D. Ma, C. W. Ong, J. Lu, and J. He, "Methodology for the evaluation of yield strength and hardening behavior of metallic materials by indentation with spherical tip," Journal of Applied Physics, vol. 94, no. 1, pp. 288-294, 2003.

[9] Y. P. Cao and J. Lu, "A new method to extract the plastic properties of metal materials from an instrumented spherical indentation loading curve," Acta Materialia, vol. 52, no. 13, pp. 4023-4032, 2004.

[10] H. Lee, J. H. Lee, and G. M. Pharr, "A numerical approach to spherical indentation techniques for material property evaluation," Journal of the Mechanics and Physics of Solids, vol. 53, no. 9, pp. 2037-2069, 2005.

[11] M. Zhao, N. Ogasawara, N. Chiba, and X. Chen, "A new approach to measure the elastic-plastic properties of bulk materials using spherical indentation," Acta Materialia, vol. 54, no. 1, pp. 23-32, 2006.

[12] P. Jiang, T. Zhang, Y. Feng, R. Yang, and N. Liang, "Determination of plastic properties by instrumented spherical indentation: expanding cavity model and similarity solution approach," Journal of Materials Research, vol. 24, no. 3, pp. 1045-1053, 2009.

[13] M.-Q. Le, "Material characterization by instrumented spherical indentation," Mechanics of Materials, vol. 46, pp. 42-56, 2012.

[14] A. Muliana, R. M. Haj-Ali, R. Steward, and A. Saxena, "Artificial neural network and finite element modeling of nanoindentation tests," Metallurgical and Materials Transactions A, vol. 33, pp. 1939-1947, 2002.

[15] N. Huber, W. Nix, and H. Gao, "Identification of elasticplastic material parameters from pyramidal indentation of thin films," Proceedings of the Royal Society of London A:
Mathematical, Physical and Engineering Sciences: The Royal Society, vol. 458, no. 2023, pp. 1593-1620, 2002.

[16] N. A. Branch, G. Subhash, N. K. Arakere, and M. A. Klecka, "Material-dependent representative plastic strain for the prediction of indentation hardness," Acta Materialia, vol. 58, no. 19, pp. 6487-6494, 2010.

[17] B. Guelorget, M. François, C. Liu, and J. Lu, "Extracting the plastic properties of metal materials from microindentation tests: experimental comparison of recently published methods," Journal of Materials Research, vol. 22, no. 6, pp. 1512-1519, 2007.

[18] E. Meyer, "Investigations of hardness testing and hardness," Phys Z, vol. 9, p. 66, 1908.

[19] D. Tabor, The Hardness of Metals, Clarendon, Oxford, UK, 1951.

[20] F. O. Sonmez and A. Demir, "Analytical relations between hardness and strain for cold formed parts," Journal of Materials Processing Technology, vol. 186, no. 1-3, pp. 163-173, 2007.

[21] O. Richmond, H. Morrison, and M. Devenpeck, "Sphere indentation with application to the Brinell hardness test," International Journal of Mechanical Sciences, vol. 16, no. 1, pp. 75-82, 1974.

[22] J. R. Matthews, "Indentation hardness and hot pressing," Acta Metallurgica, vol. 28, no. 3, pp. 311-318, 1980.

[23] E. G. Herbert, W. Oliver, and G. Pharr, "On the measurement of yield strength by spherical indentation," Philosophical Magazine, vol. 86, no. 33-35, pp. 5521-5539, 2006.

[24] B. Xu and X. Chen, "Determining engineering stress-strain curve directly from the load-depth curve of spherical indentation test," Journal of Materials Research, vol. 25, no. 12, pp. 2297-2307, 2010.

[25] H. Habbab, B. Mellor, and S. Syngellakis, "Post-yield characterisation of metals with significant pile-up through spherical indentations," Acta Materialia, vol. 54, no. 7, pp. 1965-1973, 2006.

[26] Y. Tirupataiah and G. Sundararajan, "On the constraint factor associated with the indentation of work-hardening materials with a spherical ball," Metallurgical Transactions A, vol. 22, no. 10, pp. 2375-2384, 1991.

[27] M. Yetna N’Jock, D. Chicot, X. Decoopman, J. Lesage, J. M. Ndjaka, and A. Pertuz, "Mechanical tensile properties by spherical macroindentation using an indentation strainhardening exponent," International Journal of Mechanical Sciences, vol. 75, pp. 257-264, 2013.

[28] B. Taljat, T. Zacharia, and F. Kosel, "New analytical procedure to determine stress-strain curve from spherical indentation data," International Journal of Solids and Structures, vol. 35, no. 33, pp. 4411-4426, 1998.

[29] J.-H. Ahn and D. Kwon, "Derivation of plastic stress-strain relationship from ball indentations: examination of strain definition and pileup effect," Journal of Materials Research, vol. 16, no. 11, pp. 3170-3178, 2001.

[30] Y. V. Milman, B. Galanov, and S. Chugunova, "Plasticity characteristic obtained through hardness measurement," Acta Metallurgica et Materialia, vol. 41, no. 9, pp. 2523-2532, 1993.

[31] K. Fu, L. Chang, B. Zheng, Y. Tang, and H. Wang, "On the determination of representative stress-strain relation of metallic materials using instrumented indentation," Materials \& Design, vol. 65, pp. 989-994, 2015.

[32] S. R. Kalidindi and S. Pathak, "Determination of the effective zero-point and the extraction of spherical nanoindentation stress-strain curves," Acta Materialia, vol. 56, no. 14, pp. 3523-3532, 2008. 
[33] D. K. Patel and S. R. Kalidindi, "Correlation of spherical nanoindentation stress-strain curves to simple compression stress-strain curves for elastic-plastic isotropic materials using finite element models," Acta Materialia, vol. 112, pp. 295-302, 2016.

[34] M. M. Chaudhri, "Subsurface plastic strain distribution around spherical indentations in metals," Philosophical Magazine A, vol. 74, no. 5, pp. 1213-1224, 1996.

[35] F. Yang and J. C.-M. Li, Micro And Nano Mechanical Testing of Materials And Devices, Springer, Berlin, Germany, 2008.

[36] K. L. Johnson, "The correlation of indentation experiments," Journal of the Mechanics and Physics of Solids, vol. 18, no. 2, pp. 115-126, 1970.

[37] J.-L. Bucaille, S. Stauss, E. Felder, and J. Michler, "Determination of plastic properties of metals by instrumented indentation using different sharp indenters," Acta Materialia, vol. 51, no. 6, pp. 1663-1678, 2003.

[38] E. G. Herbert, G. M. Pharr, W. C. Oliver, B. N. Lucas, and J. L. Hay, "On the measurement of stress-strain curves by spherical indentation," Thin Solid Films, vol. 398-399, pp. 331-335, 2001.

[39] H. O’Neill, Hardness Measurement of Metals and Alloys, Chapman \& Hall, London, UK, 1967.

[40] M. C. Shaw and G. J. DeSalvo, "On the plastic flow beneath a blunt axisymmetric indenter," Journal of Engineering for Industry, vol. 92, no. 2, pp. 480-492, 1970.

[41] R. T. Shield, "On the plastic flow of metals under conditions of axial symmetry," Proceedings of the royal society of London A: Mathematical, Physical and Engineering Sciences: The Royal Society, vol. 233, no. 1193, pp. 267-287, 1955.

[42] M. Scibetta, E. Lucon, R. Chaouadi, and E. van Walle, "Instrumented hardness testing using a flat punch," International Journal of Pressure Vessels and Piping, vol. 80, no. 6, pp. 345-349, 2003.

[43] G. Pharr, W. Oliver, and F. Brotzen, "On the generality of the relationship among contact stiffness, contact area, and elastic modulus during indentation," Journal of Materials Research, vol. 7, no. 3, pp. 613-617, 1992.

[44] X. Li and B. Bhushan, "A review of nanoindentation continuous stiffness measurement technique and its applications," Materials Characterization, vol. 48, no. 1, pp. 11-36, 2002.

[45] S. Vachhani, R. Doherty, and S. Kalidindi, "Effect of the continuous stiffness measurement on the mechanical properties extracted using spherical nanoindentation," Acta Materialia, vol. 61, no. 10, pp. 3744-3751, 2013.

[46] W. C. Oliver and G. M. Pharr, "Measurement of hardness and elastic modulus by instrumented indentation: advances in understanding and refinements to methodology," Journal of Materials Research, vol. 19, no. 1, pp. 3-20, 2004.

[47] Standard A. E8, "Standard test methods for tension testing of metallic materials," Annual Book of ASTM Standards, vol. 3, pp. 57-72, 2004.

[48] I. N. Sneddon, "The relation between load and penetration in the axisymmetric Boussinesq problem for a punch of arbitrary profile," International Journal of Engineering Science, vol. 3, no. 1, pp. 47-57, 1965.

[49] S. R. Kalidindi, C. A. Bronkhorst, and L. Anand, "Crystallographic texture evolution in bulk deformation processing of FCC metals," Journal of the Mechanics and Physics of Solids, vol. 40, no. 3, pp. 537-569, 1992.

[50] S. Pathak and S. R. Kalidindi, "Spherical nanoindentation stress-strain curves," Materials Science and Engineering: $R$ : Reports, vol. 91, pp. 1-36, 2015.
[51] K.-H. Kim, Y.-C. Kim, E.-c. Jeon, and D. Kwon, "Evaluation of indentation tensile properties of $\mathrm{Ti}$ alloys by considering plastic constraint effect," Materials Science and Engineering: A, vol. 528, pp. 5259-5263, 2011.

[52] C. Chang, Determining the Elastic-Plastic Properties of Metallic Materials Through Instrumented Indentation, Doctoral dissertation, Caminos, Universidad Politécnica de Madrid, Madrid, Spain, 2016. 


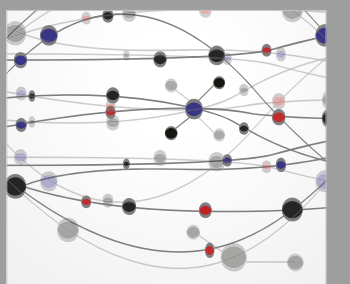

The Scientific World Journal
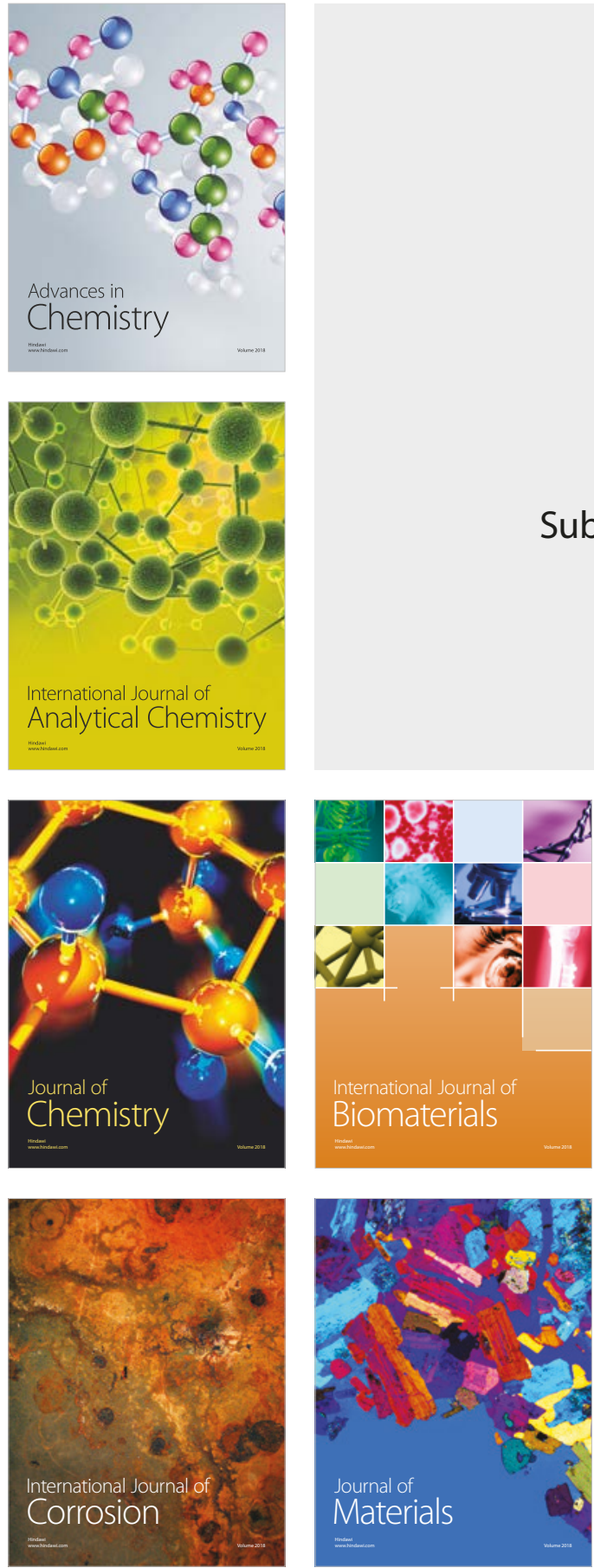

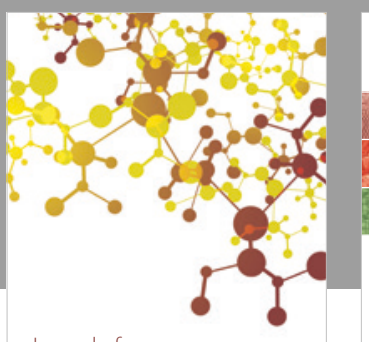

Journal of

Applied Chemistry
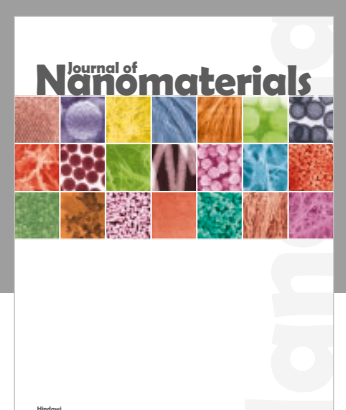

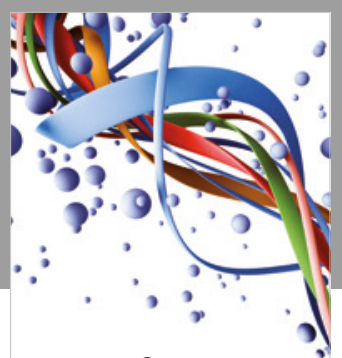

Scientifica

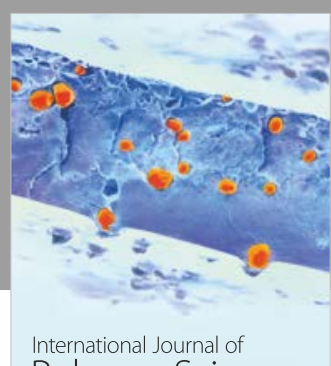

Polymer Science

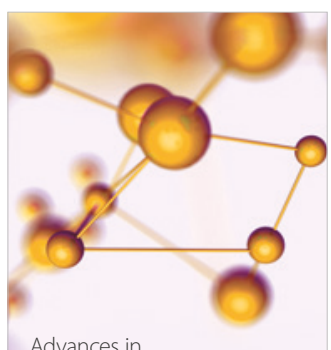

Physical Chemistry
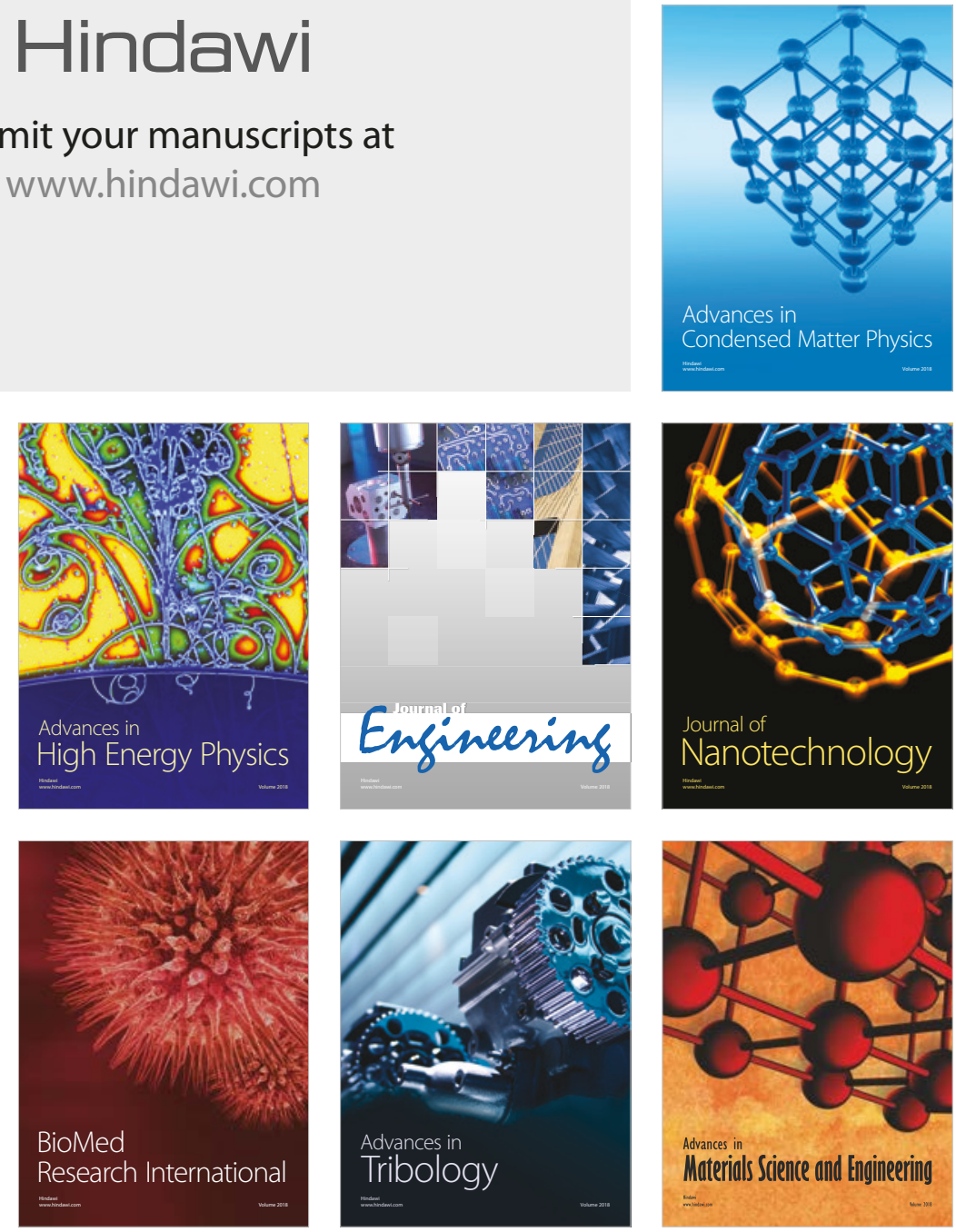